\title{
Title:
}

\section{Successful endoscopic removal of a giant duodenal bulb mass}

\section{Authors:}

Jie Zhang, Yuyong Tan, Yongjun Wang, Deliang Liu

DOI: $10.17235 /$ reed.2022.8595/2022

Link: PubMed (Epub ahead of print)

Please cite this article as:

Zhang Jie, Tan Yuyong, Wang Yongjun, Liu Deliang. Successful endoscopic removal of a giant duodenal bulb mass. Rev Esp Enferm Dig 2022. doi: 10.17235/reed.2022.8595/2022.

This is a PDF file of an unedited manuscript that has been accepted for publication. As a service to our customers we are providing this early version of the manuscript. The manuscript will undergo copyediting, typesetting, and review of the resulting proof before it is published in its final form. Please note that during the production process errors may be discovered which could affect the content, and all legal disclaimers that apply to the journal pertain. 


\section{Successful endoscopic removal of a giant duodenal bulb mass}

Jie Zhang ${ }^{1,2}$, Yuyong $\operatorname{Tan}^{1,2}$,Yongjun Wang ${ }^{1,2}$, Deliang $\operatorname{Liu}^{1,2}$

1, Department of Gastroenterology, The Second Xiangya Hospital of Central South University, Changsha, Hunan, 410011, P.R. China; 2, Research Center of Digestive Disease, Central South University, Changsha, Hunan, 410011, P.R. China

Corresponding author: Deliang Liu and Yongjun Wang, Department of Gastroenterology, The Second Xiangya Hospital, Research Center of Digestive Disease, Central South University, Changsha, Hunan, 410011, P.R. China Email: Deliangliu@csu.edu.cn, yongjunwang@csu.edu.cn

A 53-year-old man presented to our hospital for resection of a duodenal mass because of the increasing diameter. Esophagogastroduodenoscopy revealed a giant oval mass in the back wall of duodenal bulb, which was protruded to the second part of duodenum(Figure 1). Endoscopic ultrasonography (EUS) revealed a submucosal mass with heterogeneous echogenicity and regular shape(Figure 2). Eventually, the patient received endoscopic submucosal dissection (ESD) after signing informed consent. The mass was resected completely and measured $6.0 \times 4.2 \times 3.0 \mathrm{~cm}$ [Figure 3]. Histopathological examination revealed a brunner's gland adenoma. There was no complication besides minor intraoperative bleeding. Both surgery and endoscopic resection (ER) are alternative treatments for duodenal adenoma, but the best way remains controversial. Due to the thin wall, narrow cavity and plentiful vascular network of the duodenal bulb, ER is challenging because of the technical difficulty and probability of perforation and bleeding [1]. Our previous study found that ER is an effective and safe way for treating duodenal adenoma on experienced hands, and ER possesses several advantages over surgical resection for selected patients $\left[\begin{array}{ll}2,3 & 3\end{array}\right.$. In the present case, we removed the giant BGA by ESD, as far as we know, this is the largest yet removed by ER. 


\section{References}

1. Li C, Chu Y, Lv L, et al. Safety and efficacy of endoscopic resection for the treatment of duodenal subepithelial lesions. Journal of Gastrointestinal Oncology 2021;12:856-63.

2. Li C, Liang C, Wang $X$, et al. Safety and efficacy of surgical and endoscopic resection in the treatment of duodenal subepithelial lesions. Surgical Endoscopy 2021.doi: 10.1007/s00464-021-08740-3.

3. Li DF, Shi R Y, Xiong $F$, et al. Endoscopic resection in combination with ligation for the treatment of duodenal subepithelial lesions: a single-center experience. Rev Esp Enferm Dig 2021. doi: 10.17235/reed.2021.8105/2021.
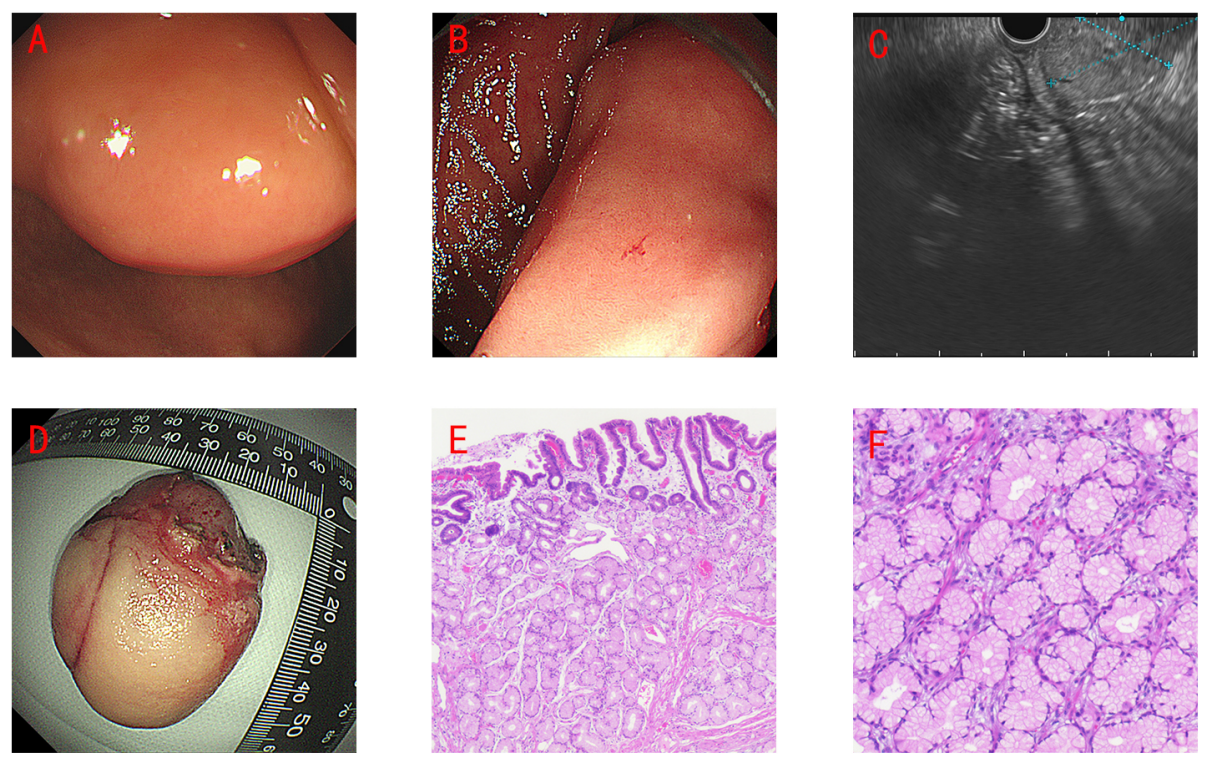

A and B. A giant duodenal bulb mass on esophagogastroduodenoscopy, protruding to the second part of duodenum.

C.Endoscopic ultrasonography revealed that the tumor originated from the submucosal layer with heterogeneous echogenicity.

D. The resected mass.

E and F. Histopathological examination revealed brunner's gland adenoma(HEx10 and $\times 40)$. 